think but neither know (explicitly or implicitly) nor care how others feel. The role of the amygdala in the development of ToM in autism and psychopathy is discussed.

06-05

\section{Theory of mind in Williams syndrome assessed using a nonverbal task}

\section{Porter}

Macquarie Centre for Cognitive Science (MACCS), Macquarie University, Sydney, Australia

Background: Williams syndrome (WS) is a rare genetic disorder associated with intellectual impairment, good verbal skills and an unusual social personality. Despite extensive interest in the area, it remains unclear whether theory of mind (ToM) abilities are impaired or intact in WS. This uncertainty most likely relates to the wide range of measures used to assess ToM abilities and the unique profile of strengths and weaknesses associated with the syndrome. The aim of this study was to examine ToM in WS using a nonverbal picture sequencing task. The picture sequencing task was selected so that the WS individuals could not rely on their good verbal skills when performing the task. The paper also aimed to further explore heterogeneity within WS and the possibility of WS subtypes.

Methods: Langdon et al.'s (1997) picture sequencing task was administered to 30 individuals with WS and to normal chronological-age-matched and mentalage-matched controls. The picture sequencing task assesses understanding of pretence, intention and false belief, while controlling for social script knowledge and physical cause-and-effect reasoning.

Results: Results indicated a specific deficit in understanding of false belief within the WS group. There was also evidence of heterogeneity in the WS group, with the false belief impairment restricted to only a particular subgroup of WS individuals identified originally by Porter and Coltheart (2005).

Conclusions: Our research, using a nonverbal task to assess ToM, indicates impaired false belief understanding in a select group of WS individuals. There are some indications that the deficits in social understanding in this group go beyond an impaired ToM.

\section{Brain Stimulation Approaches in the Treatment of Depression}

\section{Loo}

School of Psychiatry, University of New South Wales, Sydney, New South Wales, Australia

\section{Overview}

This symposium will discuss current clinical and potential future brain stimulation techniques for the treatment of depression: electroconvulsive therapy (A/Professor Loo), transcranial magnetic stimulation (Professor Mitchell), vagus nerve stimulation (Doctor Trollor), transcranial direct current stimulation, deep brain stimulation and parallels with psychosurgery (Doctor Malhi). Each talk will present an overview of the current state of the field, recent original research and possible mechanisms of action. The session will conclude with a panel discussion on similarities and differences in the neurobiological effects of these different brain stimulation approaches.

07-01

\section{ECT - future directions: a trial of the effectiveness and safety of four forms of ECT, bitemporal, bifrontal, right unilateral and right unilateral (ultrabrief pulsewidth)}

\author{
C Loo', P Sheehan², B Lyndon² \\ 'School of Psychiatry, University of New South Wales, Sydney, Australia; and \\ ${ }^{2}$ The Northside Clinic, Sydney, New South Wales, Australia
}

Background: Electroconvulsive therapy (ECT) is a highly effective treatment for depression, but its use is limited by the risk of associated cognitive side-effects. This study aimed to investigate the relative effectiveness and cognitive side-effects of ECT given in four different forms, varying in electrode placement and pulsewidth, to determine the optimal form of ECT.

Methods: Depressed in-patients referred for ECT in a private clinic in Sydney were recruited after giving informed consent. Subjects received the form of ECT prescribed by their treating psychiatrist [bitemporal at 1.5 times seizure threshold (1.5 ST), bifrontal at $1.5 \mathrm{ST}$, right unilateral at $5 \mathrm{ST}$ or right unilateral with ultrabrief pulsewidth at $6 \mathrm{ST}$ ]. Mood (MADRS) and neuropsychological functioning (digit span, Rey Auditory Verbal Learning Test, Rey figure, word 